\title{
Methylphenidate and Guanfacine Ameliorate ADHD-Like Phenotypes in Fez1-Deficient Mice
}

\author{
Akiko Sumitomo $^{a}$ Ayumi Saka ${ }^{a}$ Keisho Ueta ${ }^{a}$ Kouta Horike \\ Kazuko Hirai ${ }^{a}$ Nao J. Gamo ${ }^{\text {b }}$ Takatoshi Hikida ${ }^{a, c}$ Keiichi I. Nakayamad \\ Akira Sawa $^{b}$ Takeshi Sakurai ${ }^{a}$ Toshifumi Tomodaa, e \\ ${ }^{a}$ Department of Research and Drug Discovery, Medical Innovation Center, Kyoto University Graduate School of \\ Medicine, Kyoto, Japan; ${ }^{b}$ Department of Psychiatry, Johns Hopkins University School of Medicine, Baltimore, MD, \\ USA; ' Laboratory for Advanced Brain Functions, Institute for Protein Research, Osaka University, Osaka, Japan; \\ ${ }^{\mathrm{d}}$ Department of Molecular and Cellular Biology, Medical Institute of Bioregulation, Kyushu University, Fukuoka,

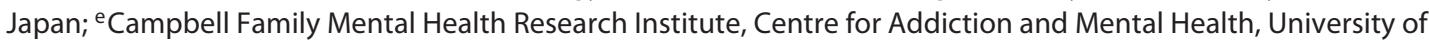 \\ Toronto, Toronto, ON, Canada
}

\section{Keywords}

FEZ1 · Neurodevelopment · Attention-deficit/hyperactivity disorder · Hyperactivity · Impulsivity · Methylphenidate . Guanfacine $\cdot$ Dopamine $\cdot$ Norepinephrine $\cdot$ Tyrosine hydroxylase

\begin{abstract}
Attention-deficit/hyperactivity disorder (ADHD) is a neurodevelopmental disorder that, while prevalent, has a stagnant track record for advances in treatment. The limited availability of animal models with appropriate face and predictive validities has hampered progress in developing novel neurobiological hypotheses and testing new therapeutic options for this condition. Here, we report that mice deficient in Fez1, a gene specifically expressed in the nervous system with documented functions in neurodevelopment, show hyperactivity and impulsivity phenotypes, which are ameliorated by administering methylphenidate (MPH) or guanfacine (GFC), two pharmacological agents used for ADHD treatment. Fez1-knockout (KO) mice show reduced expression of tyrosine hydroxylase in the midbrain and the
\end{abstract}

\section{KARGER}

(c) 2018 S. Karger AG, Basel

E-Mail karger@karger.com

www.karger.com/mnp brain stem and have reduced levels of dopamine, norepinephrine, or their metabolites in both the nucleus accumbens and the prefrontal cortex. These neurochemical changes in Fez 1-KO mice were normalized by MPH or GFC. We propose that Fez1-KO mice can be used as a model to evaluate the role of altered neurodevelopment in the manifestation of ADHD-like behavioral phenotypes, as well as to investigate the neurobiological mechanisms of existing and new pharmacotherapeutic agents for ADHD.

(C) 2018 S. Karger AG, Basel

\section{Introduction}

Attention-deficit/hyperactivity disorder (ADHD) is a prevalent neurodevelopmental condition which afflicts $5-7 \%$ of children and around $4 \%$ of adults worldwide [ 1 , 2]. Major symptoms of ADHD include inattention and/ or impulsivity and hyperactivity [3], accompanied by

Takeshi Sakurai

Medical Innovation Center, Kyoto University Graduate School of Medicine Shogoin, Sakyo-ku, 53 Kawahara-cho

Kyoto 606-8507 (Japan)

E-Mail sakurai@tk.med.kyoto-u.ac.jp

Toshifumi Tomoda

Campbell Family Mental Health Research Institute

Centre for Addiction and Mental Health, University of Toronto

250 College Street, Toronto, ON M5T 1R8 (Canada)

E-Mail ttomoda1@gmail.com 
varying degrees of cognitive, emotional, or motivational deficits, leading to major burdens at personal and socioeconomic levels [4-6]. Several clinical studies using positron emission tomography (PET) or functional magnetic resonance imaging have shown depressed dopamine (DA) activity in the caudate, nucleus accumbens (NAc), or limbic system in ADHD [7-9], whereas additional PET studies have shown that depressed DA activity is not specifically associated with ADHD but rather linked to low performance in an attention task demonstrated by both healthy individuals and those with ADHD [10]. At present, the etiological and pathophysiological mechanisms underlying this disorder remain unknown.

Methylphenidate $(\mathrm{MPH})$, a psychostimulant that blocks the DA reuptake activity of the DA transporter (DAT), is a first-choice pharmacotherapy to treat this condition, despite the risk of developing addiction and psychosis with prolonged use or overdose [11-13]. More recent efforts to develop safer pharmacotherapies for ADHD have led to the approval of guanfacine (GFC), a selective agonist of the $G_{i}$ protein-coupled $\alpha_{2 A}$ adrenergic receptor, which inhibits cAMP $\left(3^{\prime}, 5^{\prime}\right.$-cyclic adenosine monophosphate)-mediated signaling, leading to improved neurocognitive function [14]. However, a lack of appropriate animal models for $\mathrm{ADHD}$ has remained a roadblock for advancing our understanding of the pathophysiology of this condition, as well as for developing more effective treatment options.

Previous studies have led to several rodent models of ADHD-like symptoms, including spontaneously hypertensive rats (SHR) $[15,16], D A T$-knockout (KO) mice $[17,18]$, and dopamine receptor $4(D 4 R)$-KO mice [19]. In addition, a recent study has established a hyperactive line of mice through phenotypic selection performed over multiple generations [20]. Nonetheless, there are limitations associated with each model; some of these models have only face validity with limited clinical relevance or atypical psychopharmacological measures. Most of these studies have shown little consideration of neurodevelopmental aspects of ADHD etiology.

The gene encoding FEZ1 (fasciculation and elongation protein zeta-1) is specifically expressed in the nervous system, with the highest levels of expression during neurodevelopment [21]. FEZ1 is involved in several neurodevelopmental processes, such as neurite extension [21, 22], dendritic arborization [23], axonal transport [24, 25], and neuronal migration [26]. In addition, we have previously reported that Fez1-KO mice have a hyperlocomotion phenotype [27]. In the current study, we report that Fez1-KO mice show additional ADHD-related behavior- al deficits that are ameliorated by administering $\mathrm{MPH}$ or GFC. Furthermore, we evaluate catecholamine profiles and their responses to these pharmacological agents in Fez1-KO mice.

\section{Methods}

Animals

Fez1-KO mice [27] were maintained on the C57BL/6J genetic background for at least 10 generations. Fez1-KO mice and their wild-type (WT) littermate controls were used for analyses.

\section{Behavioral Assays}

Male mice were tested using a battery of behavioral assays at $6-7$ weeks of age, as previously described $[28,29]$. The personnel performing the tests were blinded to the genotype of the mice. For the pharmacological rescue experiments, MPH (Sigma; $0.5 \mathrm{mg} / \mathrm{kg}$ body weight) or GFC (Sigma; $0.1 \mathrm{mg} / \mathrm{kg}$ body weight) was dissolved in saline and intraperitoneally (i.p.) injected 30 min prior to behavioral assessment once (for acute treatment) or for 2 weeks (once daily for chronic treatment).

Locomotor Activities. Each mouse was placed at the center of an open field box $(\mathrm{W} \times \mathrm{D} \times \mathrm{H}=40 \times 40 \times 27 \mathrm{~cm}, 50 \mathrm{lux})$, and locomotor activities were tracked over $60 \mathrm{~min}$. Distance traveled $(\mathrm{cm})$ was analyzed in 5-min bins using EthoVision (Noldus).

Elevated Plus Maze. Each mouse was placed at the center of a maze $(38 \times 7 \mathrm{~cm}$ arms, $50 \mathrm{~cm}$ above the floor, 50 lux $)$ with the head facing an open arm. Total distance traveled and time spent in the open and closed arms were recorded during the 15-min session using EthoVision (Noldus).

Cliff Avoidance Reaction. Cliff avoidance reaction was evaluated as previously described [28]. Briefly, each mouse was habituated in a dark room (2.5 lux) for 30 min prior to the test, then placed on a round platform (an inverted acrylic cylinder with a diameter of $18 \mathrm{~cm}, 30 \mathrm{~cm}$ above the floor), and its behavior was video recorded for $30 \mathrm{~min}$.

\section{Quantification of Catecholamines and Their Metabolites}

To measure levels of DA, norepinephrine (NE), and their metabolites, mice ( $n=5$ per group, male, 7 weeks old) were injected (i.p.) with either saline, MPH ( $0.5 \mathrm{mg} / \mathrm{kg}$ body weight), or GFC ( 0.1 $\mathrm{mg} / \mathrm{kg}$ body weight) $30 \mathrm{~min}$ before decapitation, and the brains were quickly removed and sliced into 1 -mm-thick sections using ice-cold Brain Matrices (Ted Pella). The NAc and the prefrontal cortex (PFC) were sampled using a punch-out device (i.d. $1.2 \mathrm{~mm}$; Harris), homogenized with a sonicator for $30 \mathrm{~s}$ in $0.2 \mathrm{M}$ perchloric acid buffer ( $2 \mathrm{~mL}$ per $100 \mathrm{mg}$ tissue) containing $100 \mu \mathrm{M}$ EDTA and $100 \mu \mathrm{g} / \mathrm{mL}$ isoproterenol as an internal standard, and kept on ice for $30 \mathrm{~min}$. The homogenates were centrifuged at 15,000 rpm for 15 min at $4{ }^{\circ} \mathrm{C}$. The supernatants were collected, adjusted to a $\mathrm{pH}$ of 3 with $3 \mathrm{M} \mathrm{CH}_{3} \mathrm{COONa}$, and analyzed with a high-performance liquid chromatography system with an electrochemical detector (HTEC-500; Eicom, Kyoto, Japan). The column used for separation was an SC-5ODS (Eicom). The mobile phase was $0.1 \mathrm{M}$ sodium phosphate buffer $\left(0.1 \mathrm{M} \mathrm{NaH}_{2} \mathrm{PO}_{4}: 0.1 \mathrm{M} \mathrm{Na}_{2} \mathrm{HPO}_{4}=1,000: 160\right.$, $\mathrm{v} / \mathrm{v}), 1 \%$ methanol, $500 \mathrm{mg} / \mathrm{L}$ sodium sulfonate, and $50 \mathrm{mg} / \mathrm{L}$ EDTA. 


\section{Immunohistochemistry}

Immunohistochemistry studies were performed as previously described [30]. For immunostaining of the midbrain, mice $(n=4$ per group, male, 5-6 weeks old) were injected (i.p.) with either vehicle (saline), MPH (0.5 mg/kg body weight), or GFC $(0.1 \mathrm{mg} / \mathrm{kg}$ body weight) daily for 14 days, and perfused transcardially with $4 \%$ paraformaldehyde at 7-8 weeks of age while under anesthesia (ketamine $[100 \mathrm{mg} / \mathrm{kg}$ body weight]/xylazine $[10 \mathrm{mg} / \mathrm{kg}$ body weight] mixture, i.p.). For immunostaining of the brain stem, mice $(n=4$ per group, male) were similarly perfused at 7-8 weeks of age. The brains were then serially cut into 50 - $\mu$ m-thick coronal sections using a vibratome (VT1200 S; Leica), and the sections from one cohort of mice (littermates of WT and Fez1-KO mice treated with each drug or vehicle) were simultaneously immunostained at $4{ }^{\circ} \mathrm{C}$ for $16 \mathrm{~h}$ with an anti-tyrosine hydroxylase (TH) antibody (mouse, 1:3,000; Abcam), followed by Alexa Fluor ${ }^{\circledR} 488$-conjugated secondary antibody (Gibco) at room temperature for $1 \mathrm{~h}$, and mounted using ProLong Gold antifade reagent.

Images of one optical section ( $1 \mu \mathrm{m}$ thick) were acquired via confocal microscopy (SP8, Leica; $20 \times$ objective lens, $\mathrm{NA}=0.75$ ) from sections containing the ventral tegmental area and substantia nigra pars compacta, and from sections containing the locus coeruleus. In each mouse, 3-4 serial sections were analyzed. The levels of TH immunostaining were measured from each soma using Image $(\mathrm{NIH})$, with background levels in adjacent regions being subtracted, and the relative fluorescence intensity per soma was calculated by normalizing it by the average fluorescence intensity in WT mice, and analyzed across all sections from every mouse used.

\section{Statistical Analysis}

Behavioral assay data (means \pm standard error of the mean [SEM]) were analyzed using one-way or two-way ANOVA (analysis of variance) followed by Bonferroni post hoc tests. Catecholamine measurements and immunohistochemistry data were analyzed by Kruskal-Wallis test followed by Dunn's multiple comparison test, or by Mann-Whitney test (two-tailed). Statistical significance was marked the following way: ${ }^{*} p<0.05,{ }^{* *} p<0.01$.

\section{Results}

\section{Hyperlocomotion Is Ameliorated by MPH or GFC in}

Fez1-KO Mice

To assess baseline activities of Fez1-KO mice, we used an open field box to track their locomotor behavior for $1 \mathrm{~h}$, an experimental scheme widely used for testing ADHD-like hyperactivity in rodents [31]. The Fez1-KO mice displayed significantly higher locomotor activities than the WT littermate controls throughout the testing period (Fig. 1a), showing an approximately $30 \%$ increase in the total distance traveled (Fig. 1b), consistent with the initial characterization of this mouse line [27]. We next tested whether this hyperlocomotion phenotype was relevant to ADHD by testing the response of Fez1-KO mice to MPH or GFC, two pharmacotherapeutic agents used for ADHD treatment $[13,14]$. The hyperlocomotion phenotypes in the Fez1-KO mice were ameliorated by acute treatment with $\mathrm{MPH}$ (Fig. 1a, b) or GFC (Fig. 1c, d) $(F(55,418)=2.531, p<$ 0.0001 , for locomotion in 5 -min bins [Fig. 1a, c]; $F(5,38)=$ $4.511, p=0.0025$, for total distance traveled [Fig. 1b, d]), as well as after chronic treatment with these agents (online suppl. Fig. 1; see www.karger.com/doi/10.1159/000488081 for all online suppl. material). Note that the locomotor phenotypes in the control mice were not significantly affected by these treatments. The data suggested that Fez1-KO mice had psychopharmacological changes that might be shared with ADHD.

\section{Impulsivity Is Ameliorated by MPH or GFC in Fez1-KO Mice}

We next addressed whether Fez1-KO mice showed elevated impulsivity, another core symptom observed in ADHD. The elevated plus maze (EPM) has been used for evaluating not only emotionality but also impulsivity in several rodent models of ADHD [16, 32]. Consistent with the findings from the open field test, the Fez1-KO mice showed increased trends of locomotor activities $(F(5,44)=$ $2.818, p=0.0272$; Fig. 2a) and a significantly increased time spent in the open arms of the $\operatorname{EPM}(F(5,44)=5.404, p=$ 0.0006; Fig. 2b), suggesting that the Fez1-KO mice were less anxious or had an impulsive tendency when compared to the controls. These behavioral changes were normalized by acute treatment with GFC, but only insignificantly ameliorated with MPH (Fig. 2a, b). Note that these treatments did not affect behaviors in the controls.

To directly test impulsivity, Fez1-KO mice were assessed for cliff avoidance reaction on an elevated platform. Whereas the control mice showed high levels of cliff avoidance (i.e., low rates of dropping and jumping off the platform), the Fez1-KO mice showed progressively higher rates of dropping and jumping during the observation period $(F(58,1,218)=2.302, p<0.0001$; Fig. $2 c)$, suggesting that they showed more impulsive behaviors. We next tested whether GFC or MPH could rescue this phenotype. The lower rates of cliff avoidance reaction observed in the Fez1-KO mice were normalized by acute treatment with GFC (Fig. 2c, d). When MPH was acutely administered, the phenotype of the Fez1-KO mice was not completely normalized; however, we observed a trend for improvement, where MPH-treated Fez1-KO mice had cliff avoidance reactions higher than those of vehicle-treated $\mathrm{KO}$ mice $(p=0.1957)$ and lower than those of WT controls ( $p=0.0682$ ), showing a score in between (online suppl. Fig. 2). The data demonstrated that the impulsivity-like behaviors in Fez1-KO mice were insignificantly improved by MPH to a level comparable to that of the controls, sug- 


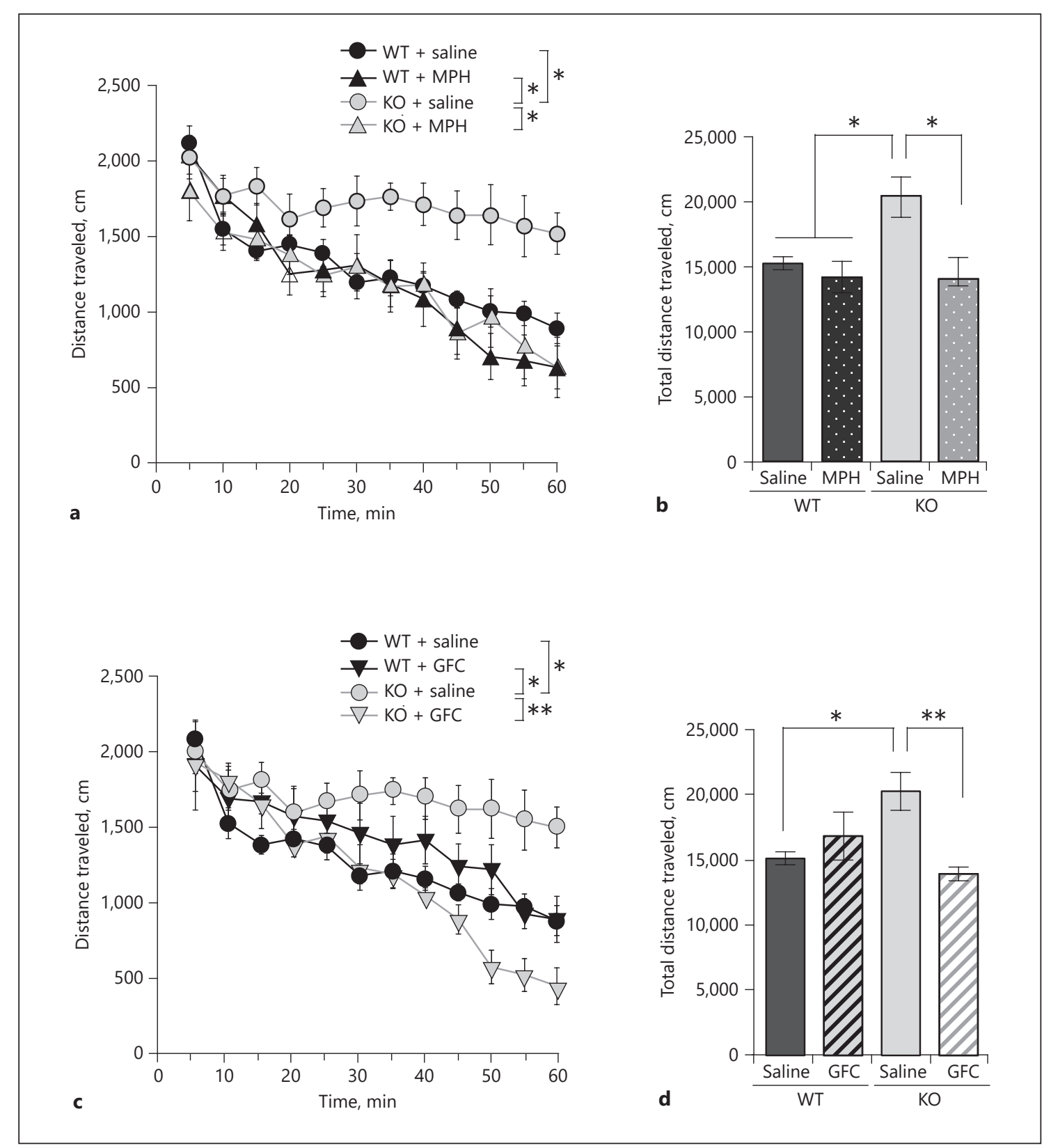

Fig. 1. Hyperlocomotion is ameliorated by methylphenidate (MPH) or guanfacine (GFC) in Fez1-knockout (KO) mice. Locomotor activities in the open field box were evaluated for wild-type (WT) control mice treated acutely with saline $(n=10), \mathrm{MPH}(0.5$ $\mathrm{mg} / \mathrm{kg}$ body weight $(n=5)$, and GFC $(0.1 \mathrm{mg} / \mathrm{kg}$ body weight $)$ $(n=5)$, and for Fez1-KO mice treated with saline $(n=8), \mathrm{MPH}$ $(n=8)$, and GFC $(n=8)$ (male, $6-7$ weeks old). a Locomotor activities shown in 5-min bins for WT mice treated with saline or $\mathrm{MPH}$, and Fez1-KO mice treated with saline or MPH. Statistical significance: $F(55,418)=2.531, p<0.0001$ (two-way ANOVA with repeated measures); ${ }^{*} p<0.05$ (Bonferroni post hoc test). b Total distance traveled $(\mathrm{cm})$ over the 60 -min test period for the mice used in a. Statistical significance: $F(5,38)=4.511, p=0.0025$ (oneway ANOVA); ${ }^{*} p<0.05$ (Bonferroni post hoc test). c Locomotor activities shown in 5-min bins for WT mice treated with saline or GFC, and Fez1-KO mice treated with saline or GFC. Statistical significance: $F(55,418)=2.531, p<0.0001$ (two-way ANOVA with repeated measures); ${ }^{*} p<0.05,{ }^{* *} p<0.01$. d Total distance traveled $(\mathrm{cm})$ over the 60-min test period for the mice used in c. Statistical significance: $F(5,38)=4.511, p=0.0025$ (one-way ANOVA); $* p<0.05,{ }^{* *} p<0.01$. 


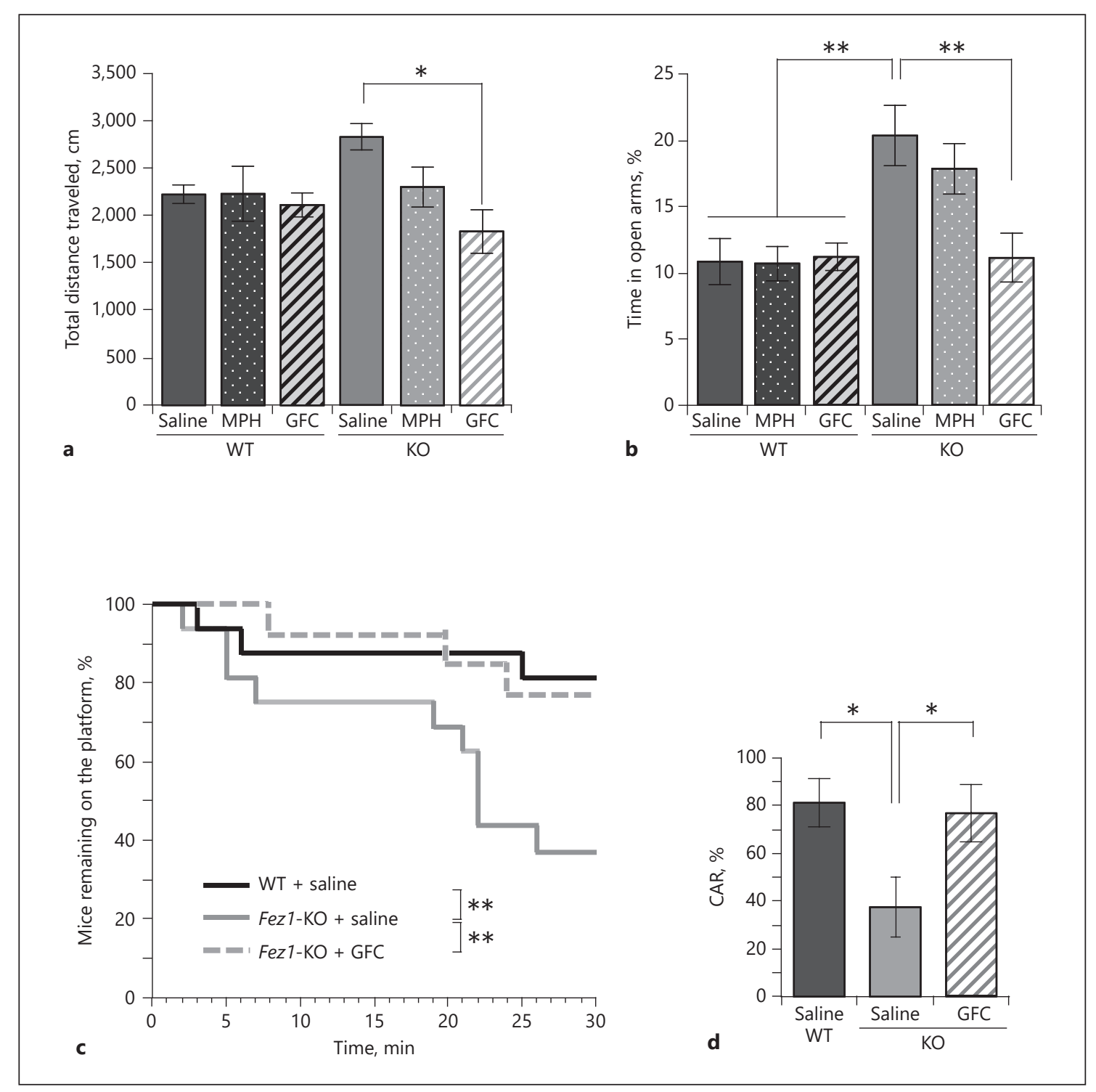

Fig. 2. Impulsivity is ameliorated by guanfacine (GFC) in Fez1knockout (KO) mice. a, b The elevated plus maze was used to test wild-type (WT) control mice treated acutely with saline $(n=11)$, methylphenidate (MPH) $(0.5 \mathrm{mg} / \mathrm{kg}$ body weight $)(n=7)$, and GFC $(0.1 \mathrm{mg} / \mathrm{kg}$ body weight $)(n=6)$, and Fez1-KO mice treated with saline $(n=8), \mathrm{MPH}(n=10)$, and GFC $(n=8)$. Statistical significance: total distance traveled $(\mathrm{cm})(\mathbf{a}), F(5,44)=2.818, p=$ 0.0272 ; time spent in open arms (\%) (b), $F(5,44)=5.404, p=$ 0.0006 (one-way ANOVA); ${ }^{*} p<0.05,{ }^{* *} p<0.01$ (Bonferroni post

gesting that MPH shows a partial rescuing effect against the impulsivity-like behaviors of Fez1-KO mice, in agreement with the results obtained with the EPM (Fig. 2a, b). These data imply that Fez1-KO mice may provide a model for elevated impulsivity that might be relevant to a core behavioral change observed in ADHD.

Predictive Validity of Fez1-KO Mice as a Model for ADHD hoc test). c Cliff avoidance behaviors were scored for $30 \mathrm{~min}$ for WT mice treated acutely with saline $(n=16)$, Fez1-KO mice treated with saline $(n=16)$, and Fez1-KO mice treated with GFC ( $n=$ 13). The percentage of mice that stayed on the platform during the test period was plotted. Statistical significance: $F(58,1,218)=$ 2.302, $p<0.0001$ (two-way ANOVA with repeated measures); ** $p<0.01$. d Percentage of cliff avoidance reaction (CAR) at the end of the test. Statistical significance: $F(2,42)=4.489, p=0.0171$ (one-way ANOVA); * $p<0.05$.

\section{Reduced Levels of Catecholamines in Fez1-KO Mice Are Rescued by MPH or GFC}

As a first step to address psychopharmacological changes in Fez1-KO brains that might underlie ADHDlike behaviors, we assessed the levels of DA and its metabolites in the NAc, a major target of dopaminergic ef- 


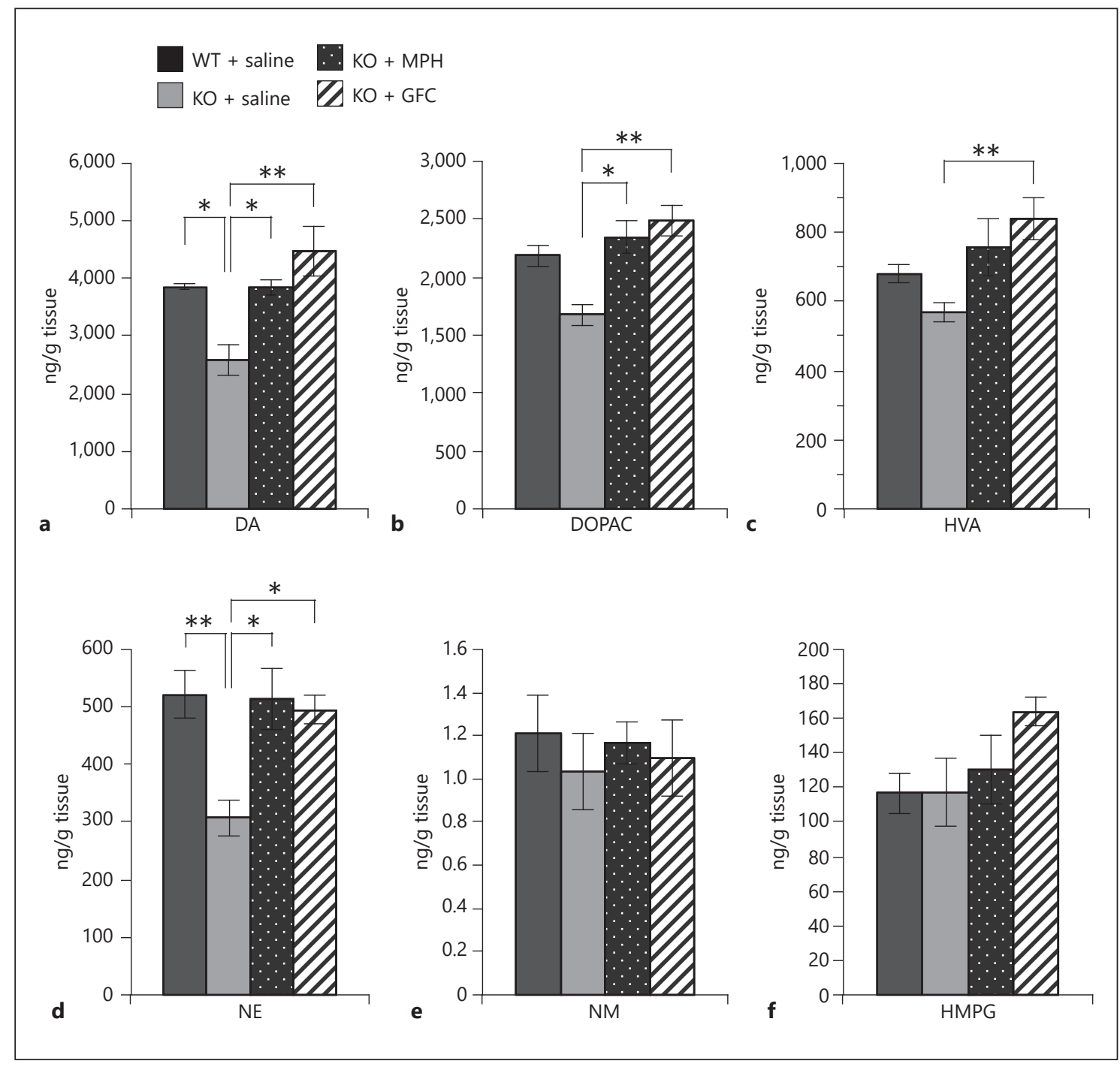

Fig. 3. Profiles of catecholamines and their metabolites in the nucleus accumbens (NAc) of Fez1-knockout (KO) mice. Levels of catecholamines and their metabolites in the NAc were measured by high-performance liquid chromatography using wild-type (WT) mice injected acutely with saline $(n=5)$, Fez1-KO mice treated with saline $(n=5)$, Fez1-KO mice treated with methylphenidate $(\mathrm{MPH})(0.5 \mathrm{mg} / \mathrm{kg}$ body weight) $(n=5)$, and Fez1-KO mice treated with guanfacine (GFC) $(0.1 \mathrm{mg} / \mathrm{kg}$ body weight) $(n=5)$ (male, 7 weeks old). a-c Levels of dopamine (DA) (a), DOPAC (b), and homovanillic acid (HVA) (c) measured in the NAc. d-f Levels of norepinephrine (NE) (d), normetanephrine (NM) (e), and HMPG (f) measured in the NAc. Statistical significance: ${ }^{*} p<0.05$, ** $p<0.01$ (Kruskal-Wallis test followed by Dunn's multiple comparison test). ferent fibers from the midbrain. Clinical studies using PET have previously reported that ADHD brains show disrupted DA neurotransmission in the NAc $[7,9,33]$. The Fez1-KO mice had significantly lower levels of DA in the NAc, which were normalized by acute administration of MPH or GFC ( $p=0.012$ by Kruskal-Wallis test) (Fig. 3a). Consistently, levels of DA metabolites (i.e., DOPAC [3,4-dihydroxyphenylacetic acid] and homova- nillic acid) were also lower in the NAc of Fez1-KO mice, and were augmented by these agents $(p=0.0118$ for DOPAC; $p=0.0157$ for homovanillic acid; by KruskalWallis test) (Fig. 3b, c). We also assessed the levels of NE and its metabolites, as GFC primarily augments NE activity [14]. The levels of NE in the NAc of Fez1-KO mice were significantly lower than those in WT mice, and they were normalized by acute administration of MPH or GFC 
Fig. 4. Reduced expression of tyrosine hydroxylase $(\mathrm{TH})$ in the midbrain is augmented by methylphenidate (MPH) or guanfacine (GFC) in Fez1-knockout (KO) mice. a Expression of TH in the ventral tegmental area and substantia nigra pars compacta in wild-type (WT) and Fez1-KO mice treated with saline, Fez1-KO mice treated with $\mathrm{MPH}(0.5 \mathrm{mg} / \mathrm{kg}$ body weight), and Fez1-KO mice treated with GFC (0.1 mg/ $\mathrm{kg}$ body weight) (daily i.p. for 2 weeks, 7-8 weeks old when perfused). Scale bar, 200 $\mu \mathrm{m}$. b Fluorescence intensities of anti-TH immunostaining per soma (80-100 somas scored per section, 3-4 serial sections analyzed per animal; $n=4$ animals used per group) above the background level were quantified by ImageJ, and normalized by the average fluorescence intensity in WT mice within each cohort (\#1, \#2, \#3, and \#4). Relative fluorescence intensities per soma from a representative section are plotted in the graph, and the overall average fluorescence intensities $( \pm$ SEM) across multiple sections per group are shown in red in the graph. Statistical significance: ** $p<0.01$ (Kruskal-Wallis test followed by Dunn's multiple comparison test).
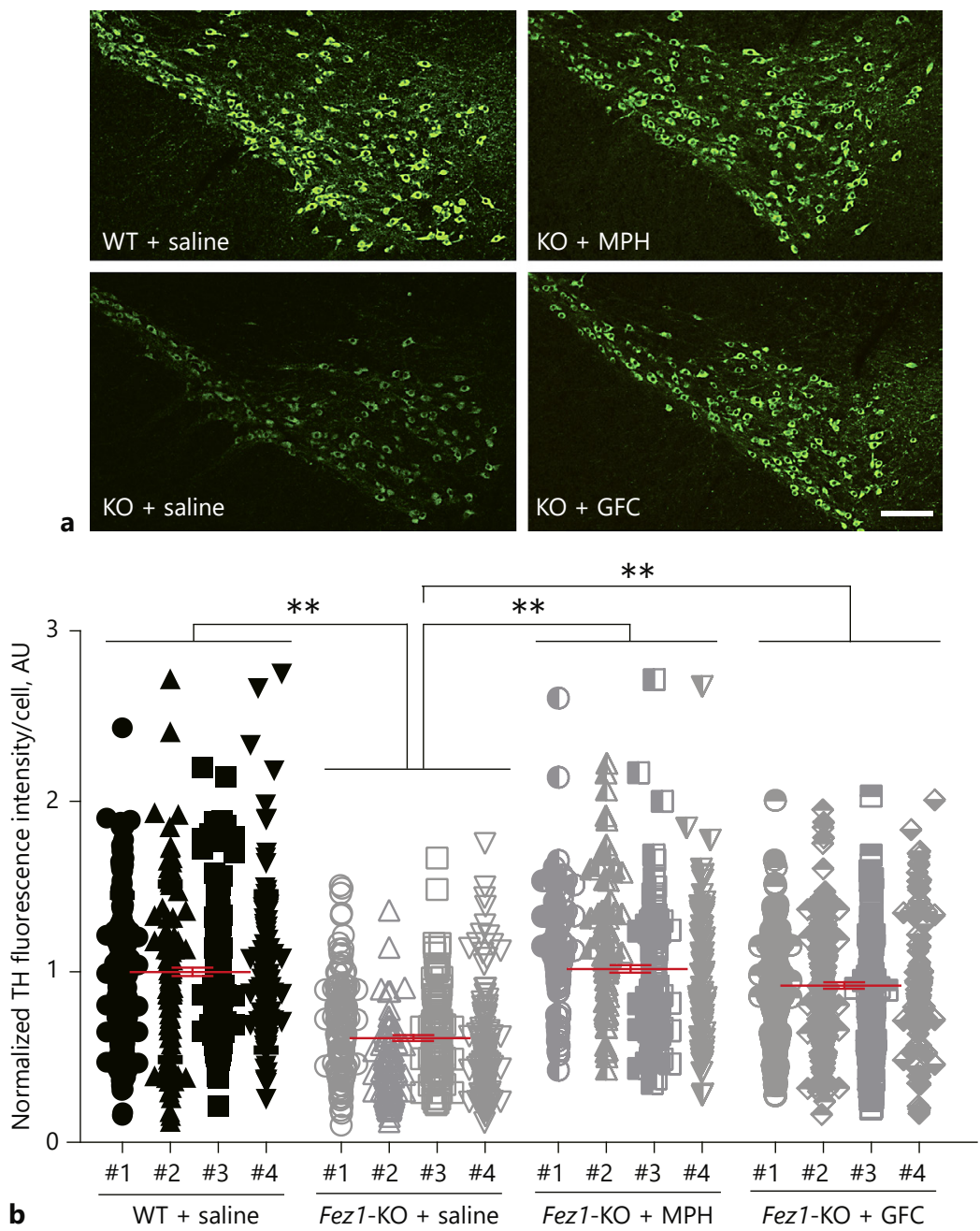

b
( $p=0.0115$ by Kruskal-Wallis test) (Fig. 3d). By contrast, the levels of its metabolites (i.e., normetanephrine and HMPG [4-hydroxy-3-methoxyphenylglycol]) were comparable between WT and Fez1-KO mice and were not significantly affected by acute administration of MPH or GFC ( $p=0.8095$ for normetanephrine; $p=0.1224$ for HMPG; by Kruskal-Wallis test) (Fig. 3e, f).

We next assessed the levels of DA, NE, and their metabolites in the PFC, another target of dopaminergic efferent fibers from the midbrain and of noradrenergic efferent fibers from the locus coeruleus in the brain stem. The Fez1-KO mice had significantly lower levels of DA in the PFC, which were normalized by acute administration of MPH or GFC ( $p=0.0051$ by Kruskal-Wallis test) (online suppl. Fig. 3a). The levels of DA metabolites showed

Predictive Validity of Fez1-KO Mice as a Model for ADHD similar trends consistent with the DA levels (online suppl. Fig. 3b, c). In addition, the levels of NE in the PFC of Fez1KO mice were significantly lower than those in WT mice and were normalized by acute administration of $\mathrm{MPH}$ or GFC ( $p=0.0038$ by Kruskal-Wallis test) (online suppl. Fig. 3d), while the levels of NE metabolites were comparable between WT and Fez1-KO mice regardless of drug administration (online suppl. Fig. 3e, f). These data together suggested that Fez1-KO mice had altered catecholamine states that might be relevant to ADHD.

\section{TH Expression Is Reduced in the Midbrain and the}

Brain Stem of Fez1-KO Mice

To investigate a biological basis for reduced catecholamine levels in Fez1-KO mice, we studied the expression 
levels of TH, a key enzyme essential for catecholamine synthesis [34], via immunohistochemistry. The levels of TH immunoreactivity in neuronal somas in the midbrain of Fez1-KO mice were significantly lower than those of controls (Fig. 4a, b). Moreover, Fez1-KO mice chronically administered MPH or GFC had TH levels significantly greater than those in Fez1-KO mice treated with vehicle alone $(1.000 \pm 0.021$ for WT mice with saline, $0.596 \pm 0.014$ for Fez1-KO mice with saline, $1.064 \pm$ 0.025 for Fez1-KO mice with MPH, and $0.886 \pm 0.017$ for Fez1-KO mice with GFC; $p<0.01$ by Kruskal-Wallis test) (Fig. 4a, b). In addition, the levels of TH immunoreactivity in the locus coeruleus in the brain stem of Fez1-KO mice were significantly lower than those of controls $(1.000 \pm 0.036$ for WT mice, $0.650 \pm 0.024$ for Fez1-KO mice; $p<0.01$ by Mann-Whitney test) (online suppl. Fig. 4). These data suggested that the reduced catecholamine levels in Fez1-KO mice could in part be due to lower expression of $\mathrm{TH}$ in the midbrain and the brain stem.

\section{Discussion}

In the present study, Fez1-KO mice showed hyperlocomotion and higher impulsivity-like phenotypes and had reduced levels of catecholamines in the NAc and the PFC along with consistent immunohistochemical changes in the midbrain and the brain stem. In addition, these changes could be ameliorated by MPH or GFC, two major pharmacotherapeutic agents used for ADHD treatment. An initial characterization of Fez1-KO mice also showed no apparent deficit in prepulse inhibition [27], a measure of sensorimotor gating. Collectively, these phenotypic and psychopharmacological alterations suggest that Fez1KO mice may serve as a new model for ADHD-like symptoms with face and predictive validity. It remains to be tested whether this mouse model also has inattention phenotypes, additional diagnostic criteria for ADHD per the DSM-5, and whether those could be normalized by $\mathrm{MPH}$ or GFC. At present, we do not know the reason for the partial rescue of the impulsivity phenotype of Fez1$\mathrm{KO}$ mice by MPH. Administering a higher dose of MPH or for a longer period of time may be necessary to achieve complete rescue, as has been tested in the clinic to determine the optimal dosing and duration of MPH for treating ADHD [35]. Nonetheless, the current data are consistent with clinical evidence that MPH is effective in alleviating both hyperactivity and impulsivity in ADHD.
Because we observed equivalent extents of significant rescue of hyperactivity in Fez1-KO mice by both acute and chronic treatments with MPH or GFC, as well as consistent increases in catecholamine contents via acute treatment, we anticipate seeing similar neurochemical changes after chronic treatment. Therefore, the behavioral rescue effect during chronic treatment could possibly be mediated by every single dose of drug treatment. Alternatively, this rescue effect via chronic treatment may be sustained by the recovery of $\mathrm{TH}$ expression achieved with the chronic treatment.

In our previous study, Fez1-KO mice showed higher sensitivity to MK-801, an N-methyl-D-aspartate receptor antagonist that elicits high levels of DA release in the NAc. Upon MK-801 injection, Fez1-KO mice showed significantly greater DA release in the NAc than control mice in microdialysis experiments [27]. Taken together with our current results, this mouse model is likely to have dysregulated catecholamine neurotransmission, which could further provide a psychopharmacological basis for the ADHD-like behavioral phenotypes observed.

Because MPH primarily blocks the activity of DAT, which is highly expressed in the caudate, NAc, and the limbic system, the primary sites of action of MPH are likely in these areas of the brain. However, the pharmacotherapeutic action of MPH could also be mediated through inhibition of the NE transporter in the PFC, leading to enhanced extracellular levels of NE and DA in the PFC and hence cognitive improvement and suppression of hyperactivity, as has been proposed to explain the therapeutic effect of MPH in DAT-KO mice [36]. By contrast, GFC primarily activates $\alpha_{2 \mathrm{~A}}$ adrenergic receptor to strengthen NE neurotransmission in the PFC, which enhances cognitive function and suppresses impulsive/ hyperactive behaviors in ADHD [37]. The mechanism by which GFC augmented DA levels in the NAc of Fez1-KO mice in the present study is currently unknown; it is possible that GFC indirectly regulates subcortical DA levels through the corticolimbic circuitry. For example, FEZ1 plays a role in anterograde transport of synaptic vesicles $[24,25]$, and could possibly regulate trafficking of DAcontaining synaptic vesicles originating from the dopaminergic neurons in the midbrain, thereby controlling $\mathrm{DA}$ release in the NAc, as previously shown [27]. It remains to be studied whether GFC could augment mesolimbic DA trafficking via activation of the corticolimbic circuitry. In this regard, it should be noted that functional and structural abnormalities in the midbrain and the NAc (e.g., altered DAT levels, size of the substantia nigra) 
have previously been reported in ADHD via clinical imaging studies $[9,33,38,39]$, as well as in a rodent model (i.e., SHR) [40].

Previous studies have provided a series of rodent models of ADHD-like symptoms, including SHR $[15,16]$, $D A T-K O$ mice $[17,18]$, and $D 4 R-K O$ mice [19], as well as a hyperactive line of mice established through phenotypic selection over multiple generations [20]. Although all these models share a hyperlocomotion phenotype, there are limitations associated with each model in terms of relevance to ADHD. For example, the SHR model was originally established as a hypertension model, and it has been difficult to determine whether the hyperactive phenotype is a primary deficit or a condition secondary to the hypertension phenotype. In addition, there are no appropriate parental control lines for SHR, making them hard to use for contemporary biomedical approaches toward drug development or pathophysiological studies [15]. $D A T$-KO mice show both face and predictive validity; however, several phenotypes, including high levels of DA (around 10 times that of controls) in the NAc and the presence of prepulse inhibition deficits, do not seem to be frequently observed in ADHD [31,41]. D4R is one of the promising risk genes identified through genome-wide association studies for ADHD, and D4R-KO mice show hyperactivity; however, this line of mice shows atypical responses to pharmacological interventions commonly used for ADHD treatment [19]. Finally, while the hyperactive line of mice developed through phenotypic selection appears to reflect the complex nature of ADHD genetics, where alteration in no single gene is attributable to the hyperactive phenotype, it makes the downstream analysis of etiology and underlying pathophysiology more difficult [20].

Although the present study has demonstrated face and predictive validity for Fez1-KO mice as a model for ADHD-like symptoms, there is currently no direct genetic evidence linking Fez1 to ADHD, except that Fez1 is mapped in proximity of an ADHD "hot spot" region in chromosome 11 [42]. Besides, it is unlikely that a single genetic alteration causes the range of symptoms associated with ADHD. Nonetheless, to the best of our knowledge, Fez1-KO mice represent the first model of ADHDlike symptoms with a potential disturbance in neurodevelopment. Previous cell biology studies have shown that FEZ1 is involved in multiple aspects of neurodevelopment, including neurite elongation [21,22], dendritic arborization [23], axonal transport [24, 25], and neuronal migration [26], by interacting with a number of proteins with documented neurobiological functions, including kinesin-1 and DISC1 (disrupted in schizophrenia-1) [2426]. Although our initial characterization of Fez1-KO mice revealed no gross anatomical changes, presumably due in part to a compensatory pathway through a family gene (Fez2) [27], we predict that either a microscopic deficit in the neuronal cytoarchitecture or a delay in neuronal development exists in Fez1-KO mice. We therefore posit that further analyses of Fez1-KO mice will facilitate dimensional studies on how disturbances in neurodevelopment may contribute to susceptibility to developing ADHD-like behavioral and psychopharmacological deficits.

\section{Acknowledgements}

This work was supported by grants from the NIH (MH-094268 Silvio O. Conte Center, MH-092443, and MH-105660), Stanley, S-R, RUSK, NARSAD, and the Maryland Stem Cell Research Fund (to A. Sawa); the Department of Defense/Congressionally Directed Medical Research Program (W81XWH-11-1-0269) and CTFDDI (to T. Tomoda); JSPS (15H01285 and 16K01948) (to T. Sakurai); JSPS (15H04275, 16H06568, and 16K14579), the Mochida Memorial Foundation for Medical and Pharmaceutical Research, International Cooperative Research Program of the Institute for Protein Research at Osaka University (ICRa-17-03), Takeda Science Foundation, and Naito Foundation (to T. Hikida); the study was also partly supported by Takeda Pharmaceutical Co. Ltd. The funders had no role in the study design and the collection, analysis, and interpretation of the data.

\section{Statement of Ethics}

Animal handling was performed in accordance with the NIH Guide for the Care and Use of Laboratory Animals, and approved by the Animal Research Committee at Kyoto University.

\section{Disclosure Statement}

We have no conflict of interest to declare.

\section{Author Contributions}

A. Sumitomo and T. Tomoda designed the study; A. Sumitomo and A. Saka conducted the experiments and statistical analysis; K. Ueta and K. Horike assisted in the behavioral experiments; K. Hirai conducted the histochemistry studies; N.J. Gamo, T. Hikida, T. Sakurai, and K.I. Nakayama provided the resources and analytical tools; N.J. Gamo edited the manuscript; A. Sawa and T. Sakurai provided advice and interpreted the data; and T. Tomoda guided the research and wrote the manuscript. 


\section{References}

$>1$ Willcutt EG: The prevalence of DSM-IV attention-deficit/hyperactivity disorder: a meta-analytic review. Neurotherapeutics 2012;9: 490-499.

$>2$ Gallo EF, Posner J: Moving towards causality in attention-deficit hyperactivity disorder: overview of neural and genetic mechanisms. Lancet Psychiatry 2016;3:555-567.

3 American Psychiatric Association: Diagnostic and Statistical Manual of Mental Disorders, ed 5 (DSM-5). Washington, American Psychiatric Association, 2013.

4 Barkley RA: Behavioral inhibition, sustained attention, and executive functions: constructing a unifying theory of ADHD. Psychol Bull 1997;121:65-94.

$>5$ Nigg JT: Is ADHD a disinhibitory disorder? Psychol Bull 2001;127:571-598.

6 Castellanos FX, Sonuga-Barke EJ, Milham MP, Tannock R: Characterizing cognition in ADHD: beyond executive dysfunction. Trends Cogn Sci 2006;10:117-123.

$>7$ Volkow ND, Wang GJ, Newcorn J, Telang F, Solanto MV, Fowler JS, Logan J, Ma Y, Schulz K, Pradhan K, Wong C, Swanson JM: Depressed dopamine activity in caudate and preliminary evidence of limbic involvement in adults with attention-deficit/hyperactivity disorder. Arch Gen Psychiatry 2007;64:932940.

$>8$ Liddle EB, Hollis C, Batty MJ, Groom MJ, Totman JJ, Liotti M, Scerif G, Liddle PF: Taskrelated default mode network modulation and inhibitory control in ADHD: effects of motivation and methylphenidate. J Child Psychol Psychiatry 2011;52:761-771.

$>9$ Volkow ND, Wang G-J, Newcorn JH, Kollins SH, Wigal TL, Telang F, Fowler JS, Goldstein RZ, Klein N, Logan J, Wong C, Swanson JM: Motivation deficit in $\mathrm{ADHD}$ is associated with dysfunction of the dopamine reward pathway. Mol Psychiatry 2011;16:1147-1154.

-10 del Campo N, Fryer TD, Hong YT, Smith R, Brichard L, Acosta-Cabronero J, Chamberlain SR, Tait R, Izquierdo D, Regenthal R, Dowson J, Suckling J, Baron JC, Aigbirhio FI, Robbins TW, Sahakian BJ, Müller U: A positron emission tomography study of nigrostriatal dopaminergic mechanisms underlying attention: implications for ADHD and its treatment. Brain 2013;136(pt 11):3252-3270.

-11 Kessler RC, Adler L, Barkley R, Biederman J, Conners CK, Demler O, Faraone SV, Greenhill LL, Howes MJ, Secnik K, Spencer T, Ustun TB, Walters EE, Zaslavsky AM: The prevalence and correlates of adult ADHD in the United States: results from the National Comorbidity Survey Replication. Am J Psychiatry 2006;163:716-723.

12 Zuvekas SH, Vitiello B, Norquist GS: Recent trends in stimulant medication use among US children. Am J Psychiatry 2006;163:579-585.
13 Berman SM, Kuczenski R, McCracken JT, London ED: Potential adverse effects of amphetamine treatment on brain and behavior: a review. Mol Psychiatry 2009;14:123-142.

14 Arnsten AF, Jin LE: Guanfacine for the treatment of cognitive disorders: a century of discoveries at Yale. Yale J Biol Med 2012;85:4558.

15 Sagvolden T, Johansen EB, Wøien G, Walaas SI, Storm-Mathisen J, Bergersen LH, Hvalby $\varnothing$, Jensen V, Aase H, Russell VA, Killeen PR, DasBanerjee T, Middleton FA, Faraone SV: The spontaneously hypertensive rat model of ADHD - the importance of selecting the appropriate reference strain. Neuropharmacology 2009;57:619-626.

16 Kishikawa Y, Kawahara Y, Yamada M, Kaneko F, Kawahara H, Nishi A: The spontaneously hypertensive rat/Izm (SHR/Izm) shows attention deficit/hyperactivity disorder-like behaviors but without impulsive behavior: therapeutic implications of low-dose methylphenidate. Behav Brain Res 2014;274: 235-242.

17 Giros B, Jaber M, Jones SR, Wightman RM, Caron MG: Hyperlocomotion and indiffer ence to cocaine and amphetamine in mice lacking the dopamine transporter. Nature 1996;379:606-612.

18 Gainetdinov RR, Wetsel WC, Jones SR, Levin $\mathrm{ED}$, Jaber M, Caron MG: Role of serotonin in the paradoxical calming effect of psychostimulants on hyperactivity. Science 1999;283: 397-401.

19 Keck TM, Suchland KL, Jimenez CC, Grandy DK: Dopamine D4 receptor deficiency in mice alters behavioral responses to anxiogenic stimuli and the psychostimulant methylphenidate. Pharmacol Biochem Behav 2013; 103:831-841.

20 Majdak P, Ossyra JR, Ossyra JM, Cobert AJ, Hofmann GC, Tse S, Panozzo B, Grogan EL, Sorokina A, Rhodes JS: A new mouse model of ADHD for medication development. Sci Rep 2016;6:39472.

21 Kuroda S, Nakagawa N, Tokunaga C, Tatematsu K, Tanizawa K: Mammalian homologue of the Caenorhabditis elegans UNC76 protein involved in axonal outgrowth is a protein kinase $\mathrm{C} \zeta$-interacting protein. J Cell Biol 1999;144:403-411.

22 Miyoshi K, Honda A, Baba K, Taniguchi M, Oono K, Fujita T, Kuroda S, Katayama T, Tohyama M: Disrupted-in-Schizophrenia 1, a candidate gene for schizophrenia, participates in neurite outgrowth. Mol Psychiatry 2003;8:685-694.

23 Watanabe Y, Khodosevich K, Monyer H: Dendrite development regulated by the schizophrenia-associated gene FEZ1 involves the ubiquitin proteasome system. Cell Rep 2014;7:552-564.
24 Toda H, Mochizuki H, Flores R 3rd, Josowitz R, Krasieva TB, LaMorte VJ, Suzuki E, Gindhart JG, Furukubo-Tokunaga K, Tomoda T: UNC-51/ATG1 kinase regulates axonal transport by mediating motor-cargo assembly. Genes Dev 2008;22:3292-3307.

25 Flores R 3rd, Hirota Y, Armstrong B, Sawa A, Tomoda T: DISC1 regulates axonal transport via a lithium-sensitive pathway. Neurosci Res 2011;71:71-77.

26 Kang E, Burdick KE, Kim JY, Duan X, Guo JU, Sailor KA, Jung DE, Ganesan S, Choi S, Pradhan D, Lu B, Avramopoulos D, Christian K, Malhotra AK, Song H, Ming GL: Interaction between FEZ1 and DISC1 in regulation of neuronal development and risk for schizophrenia. Neuron 2011;72:559-571.

-27 Sakae N, Yamasaki N, Kitaichi K, Fukuda T, Yamada M, Yoshikawa H, Hiranita T, Tatsumi Y, Kira J, Yamamoto T, Miyakawa T, Nakayama KI: Mice lacking the schizophrenia-associated protein FEZ1 manifest hyperactivity and enhanced responsiveness to psychostimulants. Hum Mol Genet 2008; 17: 3191-3203.

28 Matsuoka Y, Furuyashiki T, Yamada K, Nagai T, Bito H, Tanaka Y, Kitaoka S, Ushikubi F, Nabeshima T, Narumiya S: Prostaglandin E receptor EP1 controls impulsive behavior under stress. Proc Natl Acad Sci USA 2005;102: 16066-16071.

29 Hikida T, Jaaro-Peled H, Seshadri S, Oishi K, Hookway C, Kong S, Wu D, Xue R, Andradé M, Tankou S, Mori S, Gallagher M, Ishizuka K, Pletnikov M, Kida S, Sawa A: Dominantnegative DISC1 transgenic mice display schizophrenia-associated phenotypes detected by measures translatable to humans. Proc Natl Acad Sci USA 2007;104:14501-14506.

-30 Tomoda T, Kim JH, Zhan C, Hatten ME: Role of Unc51.1 and its binding partners in CNS axon outgrowth. Genes Dev 2004;18:541558.

31 Arime Y, Kubo Y, Sora I: Animal models of attention-deficit/hyperactivity disorder. Biol Pharm Bull 2011;34:1373-1376.

32 Pawlak CR, Karrenbauer BD, Schneider P, Ho Y-J: The elevated plus-maze test: differential psychopharmacology of anxiety-related behavior. Emot Rev 2012;4:98-115.

33 Volkow ND, Wang GJ, Kollins SH, Wigal TL, Newcorn JH, Telang F, Fowler JS, Zhu W, Logan J, Ma Y, Pradhan K, Wong C, Swanson JM: Evaluating dopamine reward pathway in ADHD: clinical implications. JAMA 2009; 302:1084-1091.

34 Kaufman S: Tyrosine hydroxylase. Adv Enzymol Relat Areas Mol Biol 1995;70:103-220. 
35 Greenhill LL, Swanson JM, Vitiello B, Davies $\mathrm{M}$, Clevenger W, Wu M, Arnold LE, Abikoff HB, Bukstein OG, Conners CK, Elliott GR, Hechtman L, Hinshaw SP, Hoza B, Jensen PS, Kraemer HC, March JS, Newcorn JH, Severe JB, Wells K, Wigal T: Impairment and deportment responses to different methylphenidate doses in children with ADHD: the MTA titration trial. J Am Acad Child Adolesc Psychiatry 2001;40:180-187.

36 Madras BK, Miller GM, Fischman AJ: The dopamine transporter and attention-deficit/hyperactivity disorder. Biol Psychiatry 2005;57: 1397-1409.

37 Arnsten AFT: The use of $\mathrm{a}-2 \mathrm{~A}$ adrenergic agonists for the treatment of attention-deficit/ hyperactivity disorder. Expert Rev Neurother 2010;10:1595-1605.
38 Jucaite A, Fernell E, Halldin C, Forssberg H, Farde L: Reduced midbrain dopamine transporter binding in male adolescents with attention-deficit/hyperactivity disorder: association between striatal dopamine markers and motor hyperactivity. Biol Psychiatry 2005;57: 229-238.

39 Romanos M, Weise D, Schliesser M, Schecklmann M, Löffler J, Warnke A, Gerlach M, Classen J, Mehler-Wex C: Structural abnormality of the substantia nigra in children with attention-deficit hyperactivity disorder. J Psychiatry Neurosci 2010;35:55-58.

40 Leo D, Sorrentino E, Volpicelli F, Eyman M, Greco D, Viggiano D, di Porzio U, PerroneCapano C: Altered midbrain dopaminergic neurotransmission during development in an animal model of ADHD. Neurosci Biobehav Rev 2003;27:661-669.
1 Barr AM, Lehmann-Masten V, Paulus M, Gainetdinov RR, Caron MG, Geyer MA: The selective serotonin-2A receptor antagonist M100907 reverses behavioral deficits in dopamine transporter knockout mice. Neuropsychopharmacology 2004;29:221-228.

- 42 Jain M, Vélez JI, Acosta MT, Palacio LG, Ba$\log \mathrm{J}$, Roessler E, Pineda D, Londoño AC, Palacio JD, Arbelaez A, Lopera F, Elia J, Hakonarson H, Seitz C, Freitag CM, Palmason $\mathrm{H}$, Meyer J, Romanos M, Walitza S, Hemminger U, Warnke A, Romanos J, Renner T, Jacob C, Lesch KP, Swanson J, Castellanos FX, Bailey-Wilson JE, Arcos-Burgos M, Muenke M: A cooperative interaction between LPHN3 and $11 \mathrm{q}$ doubles the risk for ADHD. Mol Psychiatry 2012;17:741-747. 